\title{
UM MODELO DE REGULAÇÃO FLORESTAL E SUAS IMPLICAÇÕES NA FORMULAÇÃO E SOLUÇÃ̃O DE PROBLEMAS COM RESTRIÇÕES DE RECOBRIMENTO ${ }^{1}$
}

Flávio Lopes Rodrigues ${ }^{2}$, Gilson Fernandes da Silva², Helio Garcia Leite ${ }^{3}$, Alexandre Cândido Xavier ${ }^{2}$ e José Eduardo Macedo Pezzopane ${ }^{2}$

\begin{abstract}
RESUMO - Este trabalho teve como objetivo avaliar uma estratégia utilizada para geração de alternativas de manejo na formulação e solução de problemas de planejamento florestal com restrições de recobrimento. O problema de planejamento florestal foi formulado via modelo I e modelo II, assim denominados por Johnson E Scheurman (1977), resultando em problemas de programação linear inteira com 63 e 42 alternativas de manejo, respectivamente. Conforme esperado, no problema formulado via modelo I não houve violação das restrições de recobrimento, enquanto no problema formulado via modelo II algumas unidades de manejo foram fracionadas, fato já esperado, uma vez que essa formulação não assegura a integridade das unidades de manejo. $\mathrm{Na}$ formulação via modelo II, para assegurar a integridade das unidades de manejo foi necessário reformular o problema como um problema de programação não-linear inteira, problema esse de solução ainda mais complexa do que os de programação linear inteira. As soluções eficientes dos problemas de programação não-linear inteira esbarram nas limitações de eficiências dos principais algoritmos de solução exata e na carência de aplicações dos algoritmos aproximativos na solução desse tipo de problema, a exemplo das metaeurísticas simulated annealing, busca tabu e algoritmos genéticos, tornando-se, portanto, um atrativo para pesquisas nessa área.
\end{abstract}

Palavras-chave: Regulação florestal, programação não-linear inteira e planejamento florestal.

\section{A FOREST REGULATION MODEL AND ITS IMPLICATIONS IN THE FORMULATION AND SOLUTION OF PROBLEMS WITH COVERING CONSTRAINT}

\begin{abstract}
The objective of this work was to analyze the implications of the model II, one of the strategies that have been used for generation of forest management alternatives, in the formulation and solution of problems of forest planning with covering constraint. The forest management problem was formulated via model I and model II, resulting in linear integer programming problems with 63 and 42 alternatives of management, respectively. As expected, there was no singularity constraint violation in the problem formulated via model I, while in the problem formulated via model II some management units were fractionated. This was already expected, since this formulation did not assure the integrity of the management units. To assure the integrity of the management units in the formulation via model II, it was necessary to reformulate the problem as a non-linear integer programming problem, which has a more complex solution than the linear integer programming problems. The efficient solutions of non-linear integer programming problem come up against the efficiency limitations of the main exact solution algorithms and in the lack of applications of approximative algorithms in the solution of this type of problem, such as simulated annealing metaheuristics, tabu search e genetic algorithms, becoming, therefore, attractive for this research field.
\end{abstract}

Keywords: Forest regulation, non-linear integer programming and forest planning.

\footnotetext{
${ }^{1}$ Recebido em 30.03.2005 e aceito para publicação em 05.04.2006.

${ }^{2}$ Departamento de Engenharia Rural da Universidade Federal do Espírito Santo.E-mail: <gfsilva@ cca.ufes.br)

${ }^{3}$ Departamento de Engenharia Florestal da Universidade Federal de Viçosa. E-mail: <hgleite@ ufv.br>.
} 


\section{INTRODUÇÃO}

O gerenciamento de recursos florestais tem sido auxiliado por uma variedade de ferramentas de suporte à decisão. Em diversas partes do mundo, os sistemas de suporte à decisão (SSDs) têm sido utilizados desde a década de 1970, a exemplo do Timber RAM (NAVON, 1971), Forplan (IVERSON e ALSTON, 1986), Musyc (JOHNSON et al., 1979), Planflor (TAUBENETTO, 1984), Sisflor (RODRIGUES et al., 2000), dentre outros. Em geral, os SSDs são sistemas computadorizados, interativos e amigáveis que auxilia o tomador de decisão em todas as fases do processo decisório, utilizandose de dados, modelos e conhecimento de especialista na solução de problemas malestruturados (TURBAN, 1998). Por apresentar como filosofia a integração de dados, modelos, tecnologias e recursos humanos, os SSDs contribuem para uma melhor estruturação dos problemas, melhorando o processo decisório. Dentre os vários modelos de suporte à decisão incorporados nos SSDs, os modelos de Pesquisa Operacional (PO) ocupam papel de destaque na alocação ótima dos recursos entre as diversas atividades competitivas. O processo de solução de problemas de gerenciamento florestal utilizando tais modelos requer a geração e seleção de alternativas de manejo, as quais representam as variáveis de decisão dos modelos.

Na solução de problemas utilizando os modelos de programação linear (PL) e programação inteira (PI) são usadas, basicamente, duas abordagens para a geração das alternativas de manejo, batizadas por Johnson e Scheurman (1977) como modelo I e modelo II. No modelo I, para cada unidade de manejo é gerado um conjunto de alternativas de manejo, e, uma vez dada alternativa de manejo tenha sido escolhida para dada unidade, esta permanecerá sobre tal intervenção durante o horizonte de planejamento considerado, o que permite maior identidade da unidade de manejo. Ao contrário, no modelo II regras de alocação (agrupamento e partição) de unidades de manejo quase sempre resultam na geração de menor número de alternativas de manejo para o mesmo problema, resultando, todavia, em perdas de identidades das unidades de manejo, em função das regras de alocação.

A modelagem e solução de problemas de planejamento florestal utilizando a PL tem sido feita utilizando tanto o modelo I quanto o modelo II, embora existam muito mais trabalhos utilizando a formulação via modelo I (NAUTYAL e PEARSE, 1967; NAVON,
1971; WARE e CLUTTER, 1971; TAUBE NETTO, 1984; RODRIGUEZ e MOREIRA, 1989; RODRIGUEZ et al., 1986; SILVA, 2001; RODRIGUES, 1997; RODRIGUES et al., 1998; RODRIGUES et al., 1999; RODRIGUES, 2001; RODRIGUES et al., 2004a,b). Não foi levantado na literatura pesquisada estudo utilizando o modelo II na formulação de problemas de PI. Pelo fato de que tal abordagem para um mesmo problema, em geral, resulta em menor número de variáveis, quando comparado com o modelo I, em princípio seria uma grande vantagem utilizá-la na formulação de problemas de PI, uma vez que a redução no número de variáveis inteiras resultaria em ganhos de eficiência dos algoritmos de solução dos problemas. Entretanto, as particularidades da estratégia do modelo II na formulação de alternativas de manejo pode implicar modificações drásticas no modelo de PI, resultando, por exemplo, em modelos de PI de natureza não-linear, requerendo métodos de solução menos eficientes. Este trabalho teve como principal objetivo avaliar as implicações do uso do modelo II na formulação e solução de problemas de planejamento florestal, com restrição de integridade, ou seja, problemas de programação inteira.

\section{MATERIAL E MÉTODOS}

\subsection{O problema}

Foi considerada uma área total a ser manejada de 515,00 ha, todas em regime de alto-fuste, distribuída em 10 unidades de manejo, com respectivas áreas e idades atuais, conforme Quadro 1.

Quadro 1 - Distribuição das unidades de manejo segundo suas idades e áreas

Table 1 - Distribution of management units according to their ages and areas

\begin{tabular}{ccc}
\hline $\begin{array}{c}\text { Unidade de } \\
\text { Manejo (Talhão) }\end{array}$ & $\begin{array}{c}\text { Idade (anos) } \\
\text { em } 2004\end{array}$ & Área (ha) \\
\hline 1 & 1 & 40,00 \\
2 & 2 & 50,00 \\
3 & 3 & 45,00 \\
4 & 4 & 60,00 \\
5 & 4 & 70,00 \\
6 & 5 & 50,00 \\
7 & 5 & 35,00 \\
8 & 5 & 65,00 \\
9 & 6 & 70,00 \\
10 & 6 & 30,00 \\
\hline Total & - & 515,00 \\
\hline
\end{tabular}


Para o presente estudo de caso, definiu-se um problema de planejamento florestal com as seguintes suposições:

i) As intervenções nos povoamentos incluem: corte seguido de imediata reforma via plantio, o que significa que apenas um regime de manejo (alto-fuste) será avaliado.

ii) As idades de colheita variando de 5 a 7 anos, durante o horizonte de planejamento.

iii) As unidades de manejo possuem a mesma capacidade produtiva.

iv) Apenas uma alternativa de manejo é permitida em cada talhão, o que significa que não é permitido o fracionamento dessas unidades.

v) A produção em cada período do horizonte de planejamento deverá ser de no mímino $25000,00 \mathrm{~m}^{3}$, não superando, todavia, os $35.000,00 \mathrm{~m}^{3}$.

\subsection{Fontes de dados}

Alguns dados utilizados neste trabalho são hipotéticos, o que não invalida o estudo de caso, já que o objetivo principal do estudo é apresentar um modelo alternativo para a formulação de um problema de planejamento florestal com restrições de integridade.

Para a estimativa da produção, considerou-se um modelo de povoamento total, que após ajustado resultou na equação ${ }^{4} V=e^{6,6115-58,9898 / I}$, em que V é a produção total ( $\left.\mathrm{m}^{3} / \mathrm{ha}\right)$, I é a idade (meses) e $e$ é a base dos logaritmos neperianos. Foram considerados como custos incidentes na atividade florestal: custo de plantio ( $\mathrm{R} \$ 1.500,00 / \mathrm{ha})$, custo de reforma $(\mathrm{R} \$ 1200,00 /$ ha), custo de manutenção anual ( $\mathrm{R} \$ 400,00 /$ ha) e custo de colheita $\left(\mathrm{R} \$ 15,00 / \mathrm{m}^{3}\right)$. Considerou-se o custo de manutenção ocorrente em todos os anos do horizonte de planejamento, exceto nos anos de plantio e reforma, sendo, nessa ocasião, os valores da manutenção incluídos nos custos de plantio e reforma, respectivamente. $\mathrm{O}$ custo de oportunidade e outras variáveis da análise econômica, dependentes da taxa de juros, foram calculados considerando-se uma taxa de juros de $12 \%$ ao ano. O preço da madeira foi considerado como sendo igual a $\mathrm{R} \$ 35,00 / \mathrm{m}^{3}$.

Um dos pontos cruciais na solução de um problema de planejamento florestal é a geração de alternativas de manejo. A Figura 1 ilustra uma rede de alternativas de manejo gerada segundo a lógica do modelo I. Nesse modelo, cada rota da rede representa uma alternativa de manejo para o problema. Assim, para cada uma das 10 unidades de manejo (representadas pelos quadrados) do problema é gerado um conjunto de alternativas. Uma alternativa é um conjunto de estados ou decisões que ocorrem ao longo do horizonte de planejamento. Na rede apresentada estão destacados apenas os estados de corte e reforma (CR), sendo os demais estados representados pelos arcos da rede. Em cada arco são destacadas as opções de idades (rotações), em que o povoamento poderá ser colhido e regenerado (arcos em linha cheia) ou regenerado e deixado como estoque final (arcos em linhas interrompidas). Para o problema proposto e considerando as suposições definidas, foram geradas 63 alternativas de manejo. Por exemplo, para a unidade de manejo 10 foram geradas sete alternativas, sendo uma delas a que prescreve um corte aos seis anos no início do ano zero, um segundo corte aos sete anos no início do ano sete. Essa mesma opção de manejo definirá um povoamento com um ano de idade ao final do horizonte de planejamento.

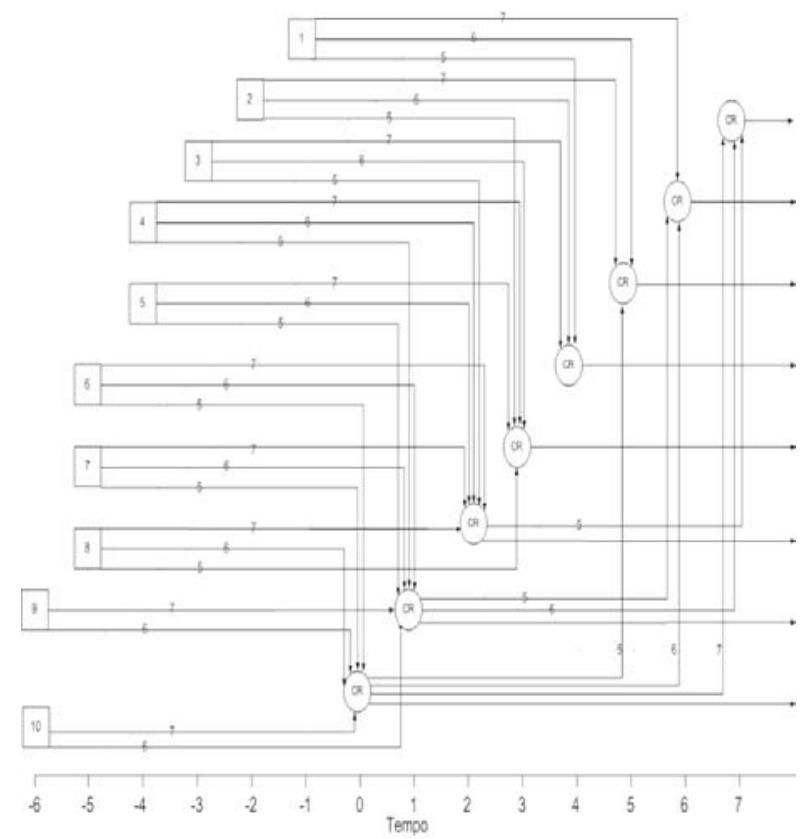

Figura 1 - Rede de manejo gerada de acordo com o modelo I. Figure 1 -Management network generated according to model I.

\footnotetext{
${ }^{4} \mathrm{R} 2$ ajustado igual a $96 \%$, para o modelo ajustado na forma logorítmica, tendo sido satisfatórias as principais suposições do modelo linear (normalidade, homocedasticidade etc).
} 
A alternativas de manejo geradas segundo a lógica do modelo II estão representadas na Figura 2. As unidades de manejo estão representadas pelos quadrados, sendo que as unidades de um a 10 representam os talhões originais, ou seja, as unidades de manejo existentes no início do horizonte de planejamento. Ao contrário do modelo I, cada arco representa uma opção de manejo para o problema. Note que as unidades de manejo com índices de 11 a 18 representam a fusão de áreas colhidas e regeneradas em um mesmo período do horizonte de planejamento. Por exemplo, a composição final da unidade de manejo 11 dependerá da estratégia adotada pelo modelo, mas poderá ser composta pela fusão de uma ou de até cinco unidades de manejo existente no início do horizonte de planejamento (unidades 6, 7, 8, 9 e 10). Essa flexibilidade do modelo II em possibilitar a criação de novas unidades de manejo é que confere a ele a principal diferença com relação ao modelo I. Neste modelo, as áreas deixadas como estoque final são representadas por uma variável de decisão (arcos com linhas interrompidas). Para o problema, formulado de acordo com a lógica do modelo II, foram geradas 42 alternativas de manejo.

\subsection{O modelo de programação inteira}

A suposição (iv) apresentada na descrição do problema estabelece uma única opção de manejo para cada uma das unidades de manejo do problema, definindo, portanto, um problema de programação inteira (PI) com as variáveis de decisão na forma binária (0-1). Formulando-se o problema mencionado e utilizando o modelo I, tem-se seguinte modelo de programação inteira:

- Função objetivo

$$
M A X \mathrm{Z}=\sum_{\mathrm{i}=1}^{\mathrm{M}} \sum_{\mathrm{j}=1}^{N} C_{i j} X_{i j}
$$

Sujeito a:

- Restrição de recobrimento

$\sum_{i=1}^{M} \sum_{j=1}^{N} X_{i j} \leq 1$

- Restrição de demanda

$\sum_{i=1}^{M} \sum_{j=1}^{N} V_{i j k} X_{i j} \leq \operatorname{Dmax}_{\mathrm{k}} \quad \mathrm{k}=\{0,1,2, \ldots, 7\}$

$\sum_{i=1}^{M} \sum_{j=1}^{N} V_{i j k} X_{i j} \geq \operatorname{Dmin}_{\mathrm{k}} \quad \mathrm{k}=\{0,1,2, \ldots, 7\}$

$\forall \mathrm{X}_{\mathrm{ij}} \in\{0,1\}$

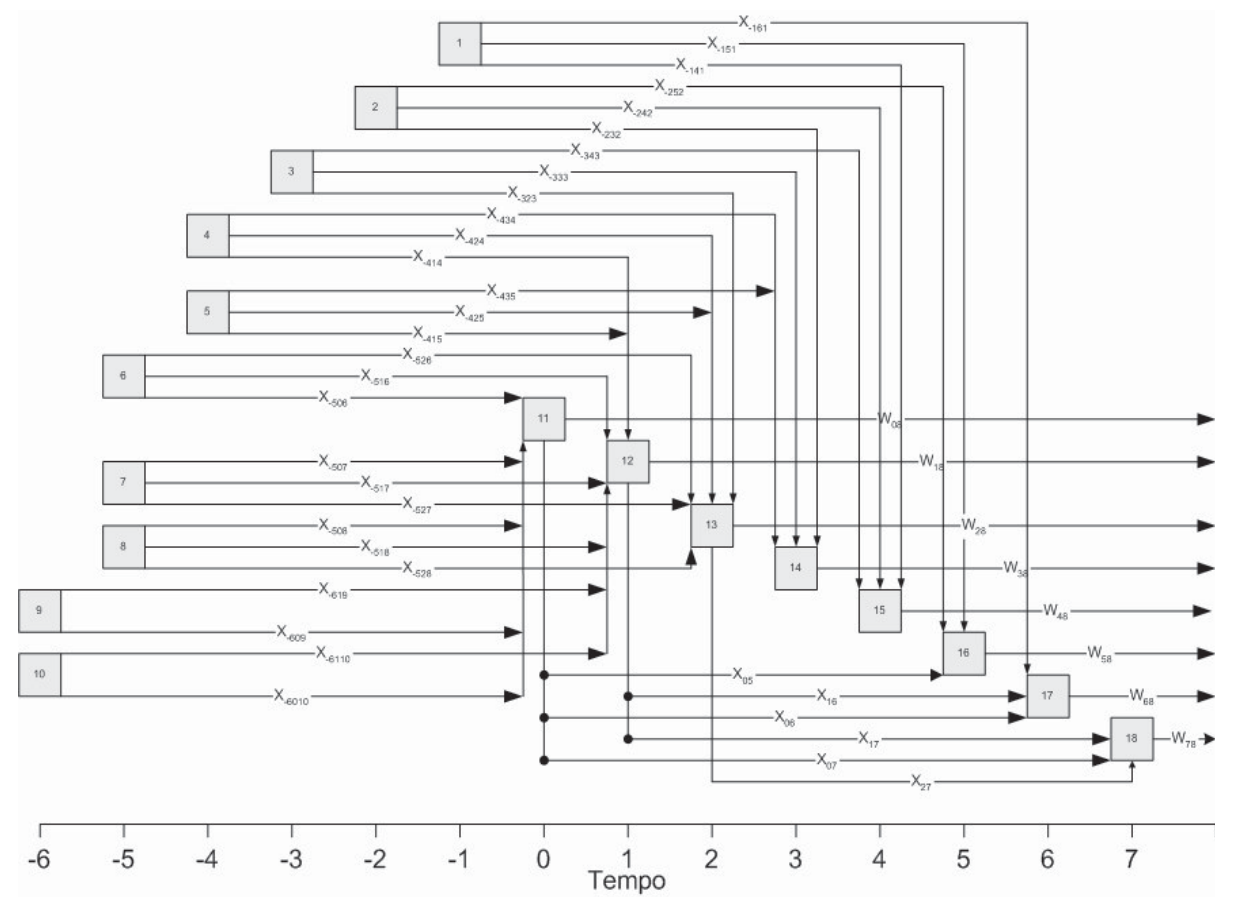

Figura 2 - Rede de manejo gerada de acordo com o modelo II.

Figure 2-Management network generated according to model model II. 
em que:

$X_{i j}=$ variável de decisão, representando a j-ésima alternativa atribuída à i-ésima unidade de manejo;

$C_{i j}=$ valor presente líquido total ${ }^{5}$ da j-ésima alternativa atribuída à i-ésima unidade de manejo;

$V_{i j k}=$ é a produção total no k-ésimo período do horizonte de planejamento da i-ésima unidade de manejo, quando atribuída pela j-ésima alternativa de manejo; e

$D_{\text {min }}$ e Dmax $\operatorname{Dm}_{k}=$ demandas $\left(\mathrm{m}^{3}\right)$ mínima e máxima de madeira, respectivamente, no k-ésimo período do horizonte de planejamento.

O mesmo problema proposto formulado via modelo II resultou no seguinte modelo de programação matemática:

- Função objetivo

$M A X \mathrm{Z}=\sum_{\mathrm{i}=-m}^{-\mathrm{im}} \sum_{\mathrm{j}=-m+R}^{-m+R} x \sum_{u=1}^{M} C_{i j u} X_{i j u}+$
$+\sum_{i=-m+R m}^{-m+R x} \sum_{j=-m+R m+R}^{H-1} Y_{i j}+\sum_{i=-m+R m+R}^{H-1} W_{i H}$

Sujeito a:

- Restrição de recobrimento

$\sum_{\mathrm{i}=-m}^{-\mathrm{i} m} \sum_{\mathrm{j}=-m+R_{m}}^{-m+R x} \sum_{u=1}^{M} X_{i j u} \leq 1$

- Restrição de reagrupamento das unidades de manejo

$\sum_{\mathrm{i}=-m}^{-\mathrm{i}_{\mathrm{m}}} \sum_{\mathrm{j}=-m+R_{m}}^{-m+R_{x}} \sum_{u=1}^{M} A_{u} X_{i j u}=\sum_{i=-m+R m}^{-m+R_{x}} \sum_{j=-m+R m+R}^{H-1} Y_{i j}+\sum_{i=-m+R m+R}^{H-1} W_{i H}$

- Restrição de demanda

$$
\begin{aligned}
& V C_{k}=\sum_{\mathrm{i}=-m}^{-\mathrm{i}_{m}} \sum_{\mathrm{j}=-m+R_{m}}^{-m+R_{x}} \sum_{u=1}^{M} V_{i j u k} X_{i j u}+\sum_{i=-m+R m}^{-m+R_{x}} \sum_{j=-m+R m+R}^{H-1} V_{i j k} Y_{i j} \geq D \min _{k} \\
& V C_{k}=\sum_{\mathrm{i}=-m}^{-\mathrm{i}_{\mathrm{m}}} \sum_{\mathrm{j}=-m+R_{m}}^{-m+R_{x}} \sum_{u=1}^{M} V_{i j u k} X_{i j u}+\sum_{i=-m+R m}^{-m+R_{x}} \sum_{j=-m+R m+R}^{H-1} V_{i j} Y_{i j k} \leq D \max _{k}
\end{aligned}
$$

$k=1,2, \ldots, \mathrm{T}$, sendo T o último período do horizonte de planejamento.
- Restrição de não-negatividade

$$
\begin{aligned}
& Y_{i j} \geq 0 \text { e } \mathrm{W}_{\mathrm{iH}} \geq 0 \\
& \forall X_{i j u} \in\{0,1\}
\end{aligned}
$$

em que:

$X_{i j u}=$ variável de decisão representando a alternativa de manejo da u-ésima unidade de manejo $(\mathrm{u}=1,2$, $\ldots, 10)$ na i-ésima classe de idade $(\mathrm{i}=-1,-2, \ldots .,-6)$, colhida no j-ésimo período do horizonte de planejamento;

$C_{i j u}=$ valor presente líquido $(\mathrm{R} \$ / \text { unidade de manejo })^{6}$ da alternativa de manejo da u-ésima unidade de manejo $(\mathrm{u}=1,2, \ldots, 10)$ na i-ésima classe de idade $(\mathrm{i}=-1$, $-2, \ldots,-6)$, colhida no j-ésimo período do horizonte de planejamento;

$Y_{i j}=$ variável de decisão representando a alternativa de manejo de uma área regenerada no i-ésimo período e colhida no j-ésimo período do horizonte de planejamento;

$C_{i j}=$ valor presente líquido ( $\left.\mathrm{R} \$ / \mathrm{ha}\right)$ da alternativa de manejo de uma área regenerada no i-ésimo período e colhida no j-ésimo período do horizonte de planejamento;

$W_{i H}=$ variável de decisão representando a alternativa de manejo de uma área regenerada no i-ésimo período e deixada como estoque ao final do horizonte de planejamento $(\mathrm{H})$;

$V_{i j u}=$ produção total $\left(\mathrm{m}^{3}\right)$ da u-ésima unidade de manejo na i-ésima classe de idade colhida no j-ésimo período do horizonte de planejamento;

$V_{i j}=$ produção $\left(\mathrm{m}^{3} / \mathrm{ha}\right)$ da i-ésima unidade de manejo colhida no j-ésimo período do horizonte de planejamento; e

$\operatorname{Dmin}_{k}$ e $\operatorname{Dmax}_{k}=$ demandas $\left(\mathrm{m}^{3}\right)$ mínima e máxima de madeira, respectivamente, no j-ésimo período do horizonte de planejamento.

\footnotetext{
${ }^{5} \mathrm{Na}$ formulação via modelo I em um problema de programação inteira binário (PI0-1) o coeficiente da função objetivo representa o retorno de toda a unidade de manejo, ao contrário da mesma formulação em problemas de programação linear (PL) onde tal coeficiente reflete uma medida por unidade de área (ha).

${ }^{6} \mathrm{Na}$ formulação via modelo I em um problema de programação inteira binário (PI0-1), o coeficiente da função objetivo representa o retorno de toda a unidade de manejo, ao contrário da mesma formulação em problemas de programação linear (PL), em que tal coeficiente reflete uma medida por unidade de área (ha).
} 


\section{RESULTADOS E DISCUSSÃO}

O problema, formulado via modelo I e modelo II, resultou em modelos de programação matemática com 63 e 42 variáveis de decisão, respectivamente. Os modelos resultantes foram solucionados pelo algoritmo branch and bound implementado no software LINDO, versão 6.0 demonstrativa. Os planos de manejo propostos para o problema, formulado via modelos I e II, estão apresentados nos Quadros 2 e 3, respectivamente. No plano de manejo sugerido pelo modelo I (Quadro 2), para cada unidade de manejo foi assinalada apenas uma variável de decisão, condição assegurada pela expressão (2), a qual define a escolha de no máximo uma alternativa de manejo para cada talhão. Assim, por exemplo, para a unidade de manejo 4 foi assinalada a variável $X_{44}$, prescrevendo colheitas nos períodos 2 e 7, com respectivas rotações de 6 e 5 anos de idade. Observe que para a unidade de manejo 6 não foi alocada nenhuma alternativa de colheita, indicando que a mesma está imune de corte durante o horizonte de planejamento sugerido. Essa condição é assegurada também pela restrição de recobrimento, uma vez que $X_{i j} \in\{0,1\}$.

Na solução sugerida pelo modelo II, com respectivo plano de manejo apresentado no Quadro 3, ocorreu o fracionamento de algumas unidades de manejo. Isso ocorreu devido à existência, nesta estrutura de modelagem, de dois grupos de unidades de manejo, aquelas referentes aos talhões originais. No exemplo, as unidades de um a 10 e as novas unidades, ou seja, aquelas obtidas por reagrupamentos ou combinações dessas unidades, ao longo do horizonte de planejamento, conforme definido pela restrição (7) do modelo II (unidades de manejo 11 a 18). Pode-se verificar, ainda, que a integridade é totalmente garantida no primeiro grupo de unidades de manejo, ou seja, inicialmente todos os talhões foram integralmente manejados com apenas uma alternativa, fato garantido pela restrição de recobrimento representada pela expressão (5), expressão que garante que todas as variáveis sejam inteiro-binárias, ou seja, $X_{i j u} \in\{0,1\}$. No entanto, a restrição de reagrupamento dos talhões (expressão 7) para formação das unidades de manejo 11 a 18, com alternativas definidas pelas variáveis de decisão $Y_{i j}$ e $W_{i H}$, resultou no fracionamento de algumas unidades de manejo, fato já esperado, uma vez que as variáveis $Y_{i j}$ e $W_{i H}$ do modelo são variáveis contínuas (equação 11). Assim, a unidade de manejo 11, com 100 ha, obtida pela fusão das unidades de manejo 9 e 10, deverá ser fracionada em duas novas unidades de manejo com 88,37 ha e 11,63 ha, colhidas nos períodos 5 e 6 , respectivamente (Quadro 3 e Figura 3). Esta estrutura resulta, na prática, no fracionamento do talhão 9 ou 10, o que é indesejável operacionalmente, já que tais unidades são, em geral, consideradas indivisíveis para efeito de manejo (corte, implantação etc.). O mesmo ocorreu com a unidade de manejo 12 (Figura 3), com 100 ha, obtida pela fusão das unidades 7 e 8 . A solução do modelo II sugere que a unidade de manejo 12 seja particionada em duas novas unidades, uma com 84,05 ha e outra com 15,95 ha, que deverão ser colhidas nos períodos 6 e 7 , respectivamente. Note que as unidades de manejo 13 , 14 e 15 foram obtidas por combinações inteiras das unidades de manejo originais. Por exemplo, a unidade 13 com 105 ha, a ser colhida no período 7 , foi obtida pela fusão das unidades 3 (45 ha) e 4 (60 ha). Essa condição assegura que as unidades originais 3 e 4 possam a ser manejadas como unidades únicas sem necessidade de fracionamento. Finalmente, as unidades de manejo 16, 17 e 18 foram obtidas por: unidade 16 ( 88,37 ha) é a maior fração da unidade 11 (88,37 ha), unidade 17 (95,67 ha) pela fusão da menor fração unidades 11 (11,62 ha) e 12 (84,05 ha), e a unidade 18 (120,95 ha), pela fusão da menor fração da unidade 12 (15,95 ha) com a unidade 13 (105 ha).

Quadro 2 - Plano de manejo proposto para o problema formulado via modelo I

Table 2 -Management planning proposed for the mixed integer programming problem formulated via model I

\begin{tabular}{|c|c|c|c|c|c|c|c|c|c|c|c|}
\hline \multirow[b]{2}{*}{ VD } & \multirow[b]{2}{*}{ Área (ha) } & \multirow[b]{2}{*}{$\mathrm{UM}$} & \multicolumn{8}{|c|}{ Rotações (anos) nos períodos } & \multirow[t]{2}{*}{ Idade final (anos) da UM } \\
\hline & & & 0 & 1 & 2 & 3 & 4 & 5 & 6 & 7 & \\
\hline$X_{11}=1$ & 40,00 & 1 & & & & & 5 & & & & 4 \\
\hline$X_{22}=1$ & 50,00 & 2 & & & & & 6 & & & & 4 \\
\hline $\mathrm{X}_{31}^{22}=1$ & 45,00 & 3 & & & 5 & & & & & 5 & 1 \\
\hline $\mathrm{X}_{44}^{31}=1$ & 60,00 & 4 & & & 6 & & & & & 5 & 1 \\
\hline$X_{56}^{44}=1$ & 70,00 & 5 & & & & 7 & & & & & 5 \\
\hline$X_{75}^{50}=1$ & 35,00 & 7 & & 6 & & & & & 5 & & 2 \\
\hline$X_{85}^{15}=1$ & 65,00 & 8 & & 6 & & & & & 5 & & 2 \\
\hline$X_{91}^{03}=1$ & 70,00 & 9 & 6 & & & & & 5 & & & 3 \\
\hline$X_{101}=1$ & 30,00 & 10 & 6 & & & & & 5 & & & 3 \\
\hline
\end{tabular}

R. Árvore, Viçosa-MG, v.30, n.5, p.769-778, 2006 
Quadro 3 - Plano de manejo proposto para o problema formulado via modelo II misto

Table 3 - Management planning proposed for the mixed integer programming problem formulated via model II

\begin{tabular}{|c|c|c|c|c|c|c|c|c|c|c|c|}
\hline \multirow[b]{2}{*}{ VD } & \multirow[b]{2}{*}{ Área (ha) } & \multirow[b]{2}{*}{ UM } & \multicolumn{6}{|c|}{ Rotações (anos) nos períodos } & \multirow[b]{2}{*}{6} & \multirow[b]{2}{*}{7} & \multirow[t]{2}{*}{ Idade final (anos) da UM } \\
\hline & & & 0 & 1 & 2 & 3 & 4 & 5 & & & \\
\hline $\mathrm{X}_{141}=1$ & 40,00 & 1 & & & & & 5 & & & & - \\
\hline $\mathrm{X}_{242}=1$ & 50,00 & 2 & & & & & 6 & & & & - \\
\hline $\mathrm{X}_{-323}=1$ & 45,00 & 3 & & & 5 & & & & & & - \\
\hline $\mathrm{X}_{424}=1$ & 60,00 & 4 & & & 6 & & & & & & - \\
\hline$X_{-435}^{-424}=1$ & 70,00 & 5 & & & & 7 & & & & & - \\
\hline $\mathrm{X}_{517}^{-453}=1$ & 35,00 & 7 & & 6 & & & & & & & - \\
\hline $\mathrm{X}_{518}=1$ & 65,00 & 8 & & 6 & & & & & & & - \\
\hline $\mathrm{X}_{-609}=1$ & 70,00 & 9 & 6 & & & & & & & & - \\
\hline$X_{-6010}=1$ & 30,00 & 10 & 6 & & & & & & & & - \\
\hline $\mathrm{Y}_{05}^{-0010}=88,37$ & 88,37 & 11 & & & & & & 5 & & & - \\
\hline$Y_{06}=11,63$ & 11,62 & 11 & & & & & & & 6 & & - \\
\hline$Y_{16}=84,05$ & 84,05 & 12 & & & & & & & 6 & & - \\
\hline$Y_{17}=15,95$ & 15,95 & 12 & & & & & & & & 7 & - \\
\hline $\mathrm{Y}_{27}=105,00$ & 105,00 & 13 & & & & & & & & 7 & - \\
\hline $\mathrm{W}_{38}=70,00$ & 70,00 & 14 & & & & & & & & & 5 \\
\hline $\mathrm{W}_{48}^{00}=90,00$ & 90,00 & 15 & & & & & & & & & 4 \\
\hline $\mathrm{W}_{58}=88,37$ & 88,37 & 16 & & & & & & & & & 3 \\
\hline $\mathrm{W}_{68}=95,67$ & 95,67 & 17 & & & & & & & & & 2 \\
\hline $\mathrm{W}_{78}=120,95$ & 120,94 & 18 & & & & & & & & & 1 \\
\hline
\end{tabular}

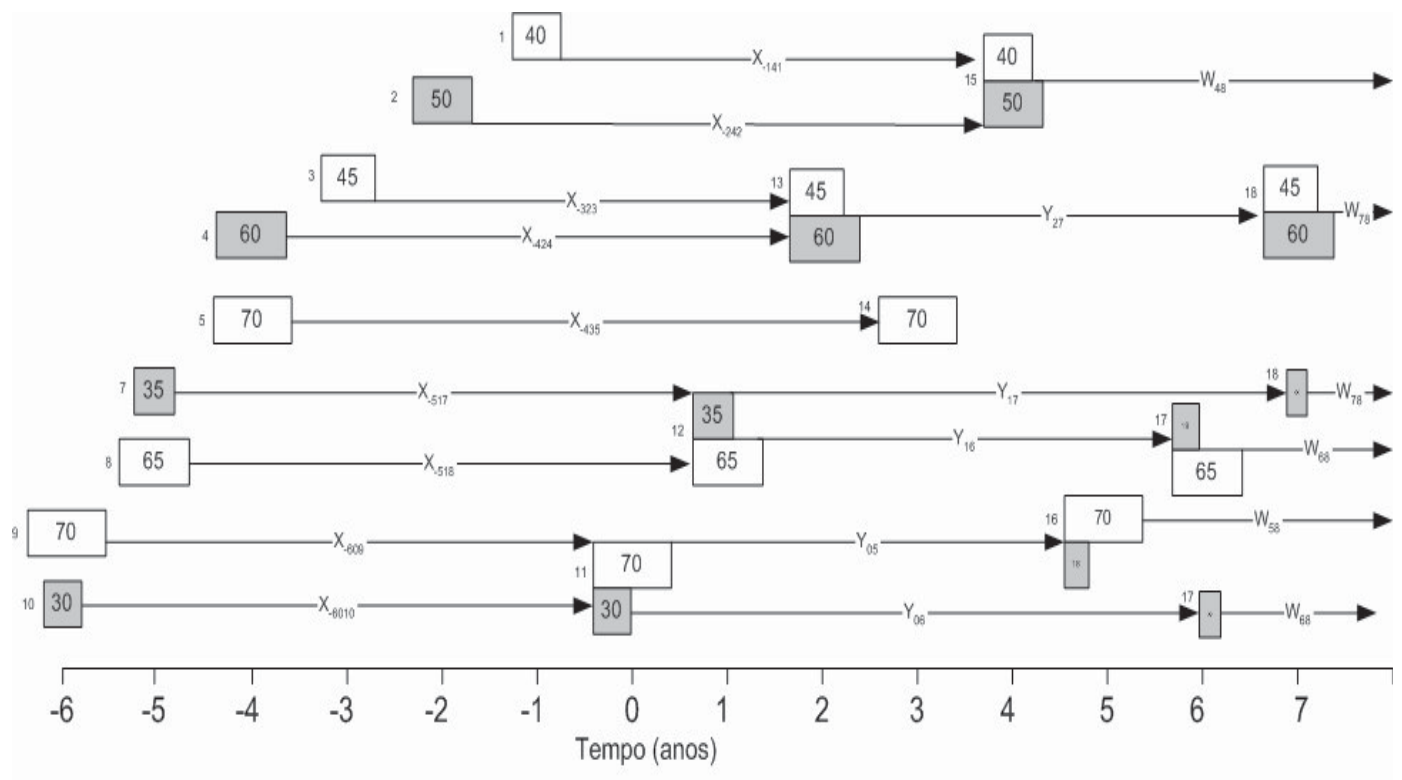

Figura 3 - Esquema de recombinação das unidades de manejo na solução proposta pelo modelo II.

Figura 3 -Scheme of recombination of management units in the solution proposed by model II.

Para resultar numa solução inteira equivalente ao modelo I, a solução do modelo II deveria corresponder ao plano de manejo proposto no Quadro 4. Observe que neste plano de manejo ocorrem apenas fusões inteiras dos talhões, o que na prática resulta na manutenção da sua integridade. O valor da função objetivo deste plano foi de $\mathrm{R} \$ 1.929 .725,00$, o que corresponde a uma redução de $0,53 \%$ em relação ao valor de $\mathrm{R} \$ 1.939 .974,00$, obtido com a solução do modelo II, apresentada no Quadro 3. 
Quadro 4 - Plano de manejo proposto para o problema formulado via modelo II inteiro

Table 4 - Management planning considered for the mixed integer programming problem formulated through model II

\begin{tabular}{|c|c|c|c|c|c|c|c|c|c|c|c|}
\hline \multirow[b]{2}{*}{ VD } & \multirow[b]{2}{*}{ Área (ha) } & \multirow[b]{2}{*}{ UM } & \multicolumn{8}{|c|}{ Rotações (anos) nos períodos } & \multirow[t]{2}{*}{ Idade final (anos) da UM } \\
\hline & & & 0 & 1 & 2 & 3 & 4 & 5 & 6 & 7 & \\
\hline$X_{141}=1$ & 40,00 & 1 & & & & & 5 & & & & - \\
\hline$X_{242}=1$ & 50,00 & 2 & & & & & 6 & & & & - \\
\hline $\mathrm{X}_{323}=1$ & 45,00 & 3 & & & 5 & & & & & & - \\
\hline$X_{424}=1$ & 60,00 & 4 & & & 6 & & & & & & - \\
\hline$X_{435}=1$ & 70,00 & 5 & & & & 7 & & & & & - \\
\hline$X_{517}=1$ & 35,00 & 7 & & 6 & & & & & & & - \\
\hline $\mathrm{X}_{518}=1$ & 65,00 & 8 & & 6 & & & & & & & - \\
\hline $\mathrm{X}_{609}=1$ & 70,00 & 9 & 6 & & & & & & & & - \\
\hline $\mathrm{X}_{6010}^{-609}=1$ & 30,00 & 10 & 6 & & & & & & & & - \\
\hline$Y_{05}^{-6010}=100,00$ & 100,00 & 11 & & & & & & 5 & & & - \\
\hline$Y_{16}=100,00$ & 100,00 & 12 & & & & & & & 6 & & - \\
\hline$Y_{27}=105,00$ & 105,00 & 13 & & & & & & & & 7 & - \\
\hline $\mathrm{W}_{38}^{27}=70,00$ & 70,00 & 14 & & & & & & & & & 5 \\
\hline $\mathrm{W}_{48}^{38}=90,00$ & 90,00 & 15 & & & & & & & & & 4 \\
\hline $\mathrm{W}_{58}^{48}=100,00$ & 100,00 & 16 & & & & & & & & & 3 \\
\hline $\mathrm{W}_{68}^{58}=100,00$ & 100,00 & 17 & & & & & & & & & 2 \\
\hline $\mathrm{W}_{78}=105,00$ & 105,00 & 18 & & & & & & & & & 1 \\
\hline
\end{tabular}

No Quadro 5 estão apresentados os volumes e as áreas de colheitas anuais obtidos com a solução do problema formulado via modelos I e II. Note que as diferenças de valores ocorrem nos três últimos períodos, resultado das diferentes estratégias de solução adotada por cada um dos modelos.

Observa-se, pelos resultados anteriores, que os planos de manejo resultantes apresentados nos Quadros 2 e 3 diferem entre si, o que já era esperado, uma vez que no modelo I foi possível a definição de todas as 63 variáveis do problema como variáveis inteiras (binárias). No entanto, no modelo II apenas um subconjunto de 28 variáveis foram declaras como inteiras binárias, enquanto as outras 14 variáveis foram definidas como contínuas, resultando, portanto, em um problema de programação inteira mista. Essa condição não assegura a integridade dos talhões. Portanto, para assegurar a integridade dos talhões, utilizando a formulação via modelo II, é necessário reformular o problema, alterando a restrição (7) para:

$$
\begin{aligned}
& \sum_{\mathrm{i}=-m}^{-\mathrm{i}_{\mathrm{m}}} \sum_{\mathrm{j}=-m+R_{m}}^{-m+R_{x}} \sum_{u=1}^{M} A_{u} X_{i j u}=\sum_{i=-m+R m}^{-m+R_{x}} \sum_{j=-m+R m+R}^{H-1} Y_{i j} Z_{i j}+\sum_{i=-m+R m+R}^{H-1} W_{i H} Z_{i H} \\
& \sum_{i=-R m}^{-m+R x} \sum_{j=-m+R m+R}^{H-1} Z_{i j}+\sum_{i=-m+R m+R}^{H-1} Z_{i H} \leq 1 \forall Z_{\mathrm{ij}} \mathrm{e} Z_{\mathrm{iH}} \in\{0,1\}
\end{aligned}
$$

A restrição (13) só é necessária quando o lado direito de (12) possuir pelo menos duas variáveis, o que pode resultar em um número de variáveis inteiras binárias inferior ao total de variáveis do problema.
Por exemplo, de acordo com a expressão (7), a restrição que define a criação de uma nova unidade de manejo no período zero (unidade 11) deve ser escrita como:

$50 X_{\_506}+35 X_{-507}+65 X_{-508}+70 X_{609}+30 X_{6010}-Y_{05}$ $-\mathrm{Y}_{06}-\mathrm{Y}_{07}-\overline{\mathrm{W}}_{08}=0$

Essa condição não assegura a integridade das unidades de manejo, uma vez que tanto $\mathrm{Y}_{\mathrm{ij}}$ quanto $\mathrm{W}_{\mathrm{iH}}$ são variáveis contínuas, podendo resultar no assinalamento de mais de uma variável (alternativa de manejo) para cada unidade de manejo. Para garantir a integridade das unidades de manejo, a expressão acima (reescrita de acordo com as expressões 12 e 13), deve ser escrita como:

Quadro 5 - Volumes e áreas de corte anuais para o problema formulado via modelos I e II

Table 5-Annual volumes and cutting areas for the mixed integer programming problem formulated via models I and II

\begin{tabular}{cccccc}
\hline \multirow{2}{*}{$\begin{array}{c}\text { Período } \\
\text { anual }\end{array}$} & \multicolumn{2}{c}{$\begin{array}{c}\text { Volume de corte } \\
\text { anual }\left(\mathrm{m}^{3}\right)\end{array}$} & & \multicolumn{2}{c}{$\begin{array}{c}\text { Área de corte } \\
\text { anual }\end{array}$} \\
\cline { 2 - 3 } \cline { 5 - 6 } & Mod. I & Mod. II & & Mod. I & Mod. II \\
\hline 0 & 33231,60 & 33231,60 & & 100,00 & 100,00 \\
1 & 33231,60 & 33231,60 & & 100,00 & 100,00 \\
2 & 32668,31 & 32668,31 & & 105,00 & 105,00 \\
3 & 26098,69 & 26098,69 & & 70,00 & 70,00 \\
4 & 27930,78 & 27930,78 & & 90,00 & 90,00 \\
5 & 28287,44 & 25000,00 & & 100,00 & 88,37 \\
6 & 28287,44 & 27638,42 & & 100,00 & 95,67 \\
7 & $29,701,82$ & 35000,00 & & 105,00 & 120,94 \\
\hline
\end{tabular}




$$
\begin{aligned}
& 50 X_{-506}+35 X_{-507}+65 X_{-508}+70 X_{-609}+30 X_{-6010}-Y_{05} Z_{05}-Y_{06} Z_{06} \\
& -Y_{07}^{-} Z_{07}-W_{08}^{-} Z_{08}=0 \\
& Z_{05}+Z_{06}+Z_{07}+Z_{08}=1 \ll Z_{i j} \text { e } Z_{i H} \in\{0,1\}
\end{aligned}
$$

Disso resulta que o novo modelo de programação matemática, formulado de acordo com a estratégia do modelo II, é um modelo de programação não-linear com variáveis inteiras e variáveis contínuas, portanto um modelo de programação não-linear inteira mista (PNLIM), com 42 variáveis, sendo 37 variáveis inteiras binárias e cinco variáveis contínuas.

A solução do modelo de PNLIM é apresentada no Quadro 4. Observe que, de acordo com esse plano de manejo, é garantida a integridade dos talhões, a exemplo do que ocorreu com a solução do modelo de Programação Linear Inteira (PLI), formulado de acordo com a estratégia do modelo I. Apesar de as soluções terem sido a mesma, existem diferenças marcantes entre as duas abordagens. Apesar do modelo de PNLIM possuir menor número de variáveis inteiras binárias, em geral os algoritmos de solução desses modelos são menos eficientes. Outro aspecto refere-se à solução dos modelos de PLI utilizando métodos aproximativos, como Algoritmos Genéticos (AG), Busca Tabu (BT) e Simulated Annealing (SA), dentre outras técnicas heurísticas, conforme encontrados em Laroze et al. (1997), Laroze (1999), Boston e Bettinguer (1999), Nobre (1999), Ignácio et al. (2000), Rodrigues (2001), Rodrigues et al. (2003) e Rodrigues et al. $\left(2004^{\mathrm{a}}, \mathrm{b}\right)$. Todos esses trabalhos desenvolveram as estratégias das respectivas heurísticas, utilizando o modelo I como estratégia de modelagem. Considerando que para um mesmo problema, em geral, o modelo II resulta em um menor número de variáveis de decisão, em princípio haveria uma vantagem da utilização desta abordagem, uma vez que a eficiência de solução de um problema é, em geral, maior para problemas com menor número de variáveis. Entretanto, a formulação via modelo II resulta em um problema de PNLIM, requerendo outras estratégias de construção dos métodos aproximativos, constituindo-se em um campo fértil para investigação em problemas de planejamento florestal, fato este ainda não estudado.

\section{CONCLUSÕES}

Este trabalho avaliou as implicações do uso do modelo II, assim batizado por Johnson e Scheurman (1977), na formulação e solução de problemas de planejamento florestal. Após comparar as estratégias de formulação e solução com o modelo I, assim definido pelos mesmos autores, chegou-se às seguintes conclusões:

A formulação via modelo II resulta em problemas de programação matemática com menor número de variáveis quando comparado com o modelo I.

Para garantir a integridade das unidades de manejo utilizando a formulação via modelo II é necessário definir restrições de natureza não-linear, resultando em problemas de programação não-linear inteira, sendo esses problemas de solução ainda mais complexa, em comparação com os problemas de programação linear inteira, formulados via modelo I.

A solução dos problemas de programação nãolinear inteira esbarra na baixa eficiência dos principais algoritmos de solução exata. Outra limitação na solução desses problemas é a carência de algoritmos aproximativos desenvolvidos, a exemplo do que já ocorre nos problemas de programação linear inteira formulados via modelo I, constituindo-se, portanto, em um campo fértil para futuras pesquisas.

\section{REFERÊNCIAS BIBLIOGRÁFICAS}

BOSTON, K.; BETTINGER, P. An analysis of monte carlo integer programming, simulated annealing, and tabu search heuristics for solving spatial harvest scheduling problems. Forest Science, v. 45, n. 2, p. 292-301, 1999.

IGNACIO, A.A.V.; FERREIRA FILHO, V.J.M.; GALVÃO, R.D. Métodos heurísticos num entorno paralelo. In: SIMPÓSIO BRASILEIRO DE PESQUISA OPERACIONAL, 32., 2000, Viçosa. Anais... Viçosa, MG: Universidade Federal de Viçosa, 2000. p. 769-788.

IVERSON, D.C.; ALSTON, R.M. The genesis of FORPLAN a historical and analytical review of Forest Service planning models. Washington: USDA Forest Service, 1986. 25 p. (General Technical Report, INT-214).

JOHNSON, K. N., SCHEURMAN, H. L. Techniques for prescribing optimal timber harvest and investment under different objectives - discussion and synthesis. Forest Science, v. 18, n. 1, p. 1-31, 1977.

R. Árvore, Viçosa-MG, v.30, n.5, p.769-778, 2006 
JOHNSON, K.N.; JONES, D.; DANIEL, B. A user's guide to multiple use sustained yield scheduling calculation (MUSYC). Fort Collins: Department of Agriculture, Forest Service, Timber Management, 1979. 242 p.

LAROZE, A.J. A linear programming, tabu search method for solving forest-level bucking optimization problems. Forest Science, v. 45, n. 1, p. $108-116,1999$.

LAROZE, A.J.; GREBER, B.J. Using Tabu search to generate stand-level, rule-based bucking patterns. Forest Science, v. 43, n. 2, p. 157-169, 1997.

NAUTIYAL, J.C.; PEARSE, P.H. Optimizing the conversion to sustained-yield - a programming solution. Forest Science, v. 13, p. 131-139, 1967.

NAVON, D.I. Timber RAM ... a long-range planning method for commercial timber lands under multiple-use management. Berkeley: Department of Agriculture, Forest Service, Pacific Southwest Forest and Range Experiment Station, 1971. 22 p. (USDA. Forest Service, Res. paper PNW - 70)

NOBRE, S.R. A heurística da Razão-R aplicada a problemas de gestão florestal. 1999. 128 f. Dissertação (Mestrado em Administração Rural) - Universidade Federal de Lavras, Lavras, 1999.

RODRIGUES, F.L. Metaheurística e sistema de suporte à decisão no gerenciamento de recursos florestais. 2001. 225 f. Tese (Doutorado em Manejo Florestal) - Universidade Federal de Viçosa, Viçosa, MG, 2001.

\section{RODRIGUES, F.L. Regulação de florestas} equiâneas utilizando programação linear. 1997. 109 f. Dissertação (Mestrado em Manejo Florestal) - Universidade Federal de Viçosa, Viçosa, MG, 1997.

RODRIGUES, F.L.; LEITE, H.G.; ALVES, J.M. Sysflor - um sistema de suporte à decisão em planejamento florestal. In: SIMPÓSIO

BRASILEIRO DE PESQUISA OPERACIONAL, 32., 2000, Viçosa. Anais... Viçosa, MG: Universidade Federal de Viçosa, 2000. p. 974-995.

R. Árvore, Viçosa-MG, v.30, n.5, p.769-778, 2006
RODRIGUES, F.L. et al. Metaheurística Simulated annealing para a solução de problema de planejamento florestal com restrições de inteireza. Revista Árvore, v. 28, n. 2. p. 256-271, 2004a.

RODRIGUES, F.L. et al. Solução de problema de planejamento florestal com restrições de inteireza utilizando busca tabu. Revista Árvore, v. 27, n. 5, p. 701-713, 2003.

RODRIGUES, F.L. et al. Solução de problema de planejamento florestal com restrições de inteireza utilizando algoritmo genético. Revista Árvore, v. 27 , n. 2. p. 311-330, 2004 b.

RODRIGUES. F.L. et al. Determinação de estratégias ótimas de reforma, condução da brotação e compra de terras, utilizando programação linear. Revista Árvore, v. 23, n. 2, p. 169-186, 1999.

RODRIGUES. F.L. et al. Regulação de florestas equiâneas utilizando programação linear: uma aplicação da teoria do modelo II. R. Árvore, v. 22, n. 2, p. 193-213, 1998.

RODRIGUEZ, L.C.E. et al. Programação Linear no planejamento florestal: uma aplicação prática. Silvicultura, v. 11, n. 41, p. 163-168, 1986.

RODRIGUEZ, L.C.E.; MOREIRA, R.M.

Gerenciamento de florestas de Eucalyptus com modelos de programação linear. IPEF, v. 6, n. 19, p. 1-15, 1989. (Série Técnica).

SILVA, G.F. Problemas no uso de programação matemática e simulação em regulação florestal. 2001. 89 f. Tese (Doutorado em Manejo Florestal) - Universidade Federal de Viçosa, Viçosa, MG, 2001.

TAUBE NETTO, M. Um modelo de programação linear para planejamento de florestas de eucalipto. Pesquisa Operacional, v. 4, n. 1, p. 19-39, 1984.

TURBAN, E; ARONSON, J.E. Decision support systems and intelligent systems. New Jersey: Prentice-Hall, 1998. 890p.

WARE, G.O.; CLUTTER, J.L. A mathematical programming system for the management of industrial forests. Forest Science, v. 17, n. 3, p. 428-445, 1971. 